BULLETIN Bulletin hispanique

HispaniquE Université Michel de Montaigne Bordeaux

$116-2$ | 2014

Référentialité/autoréférentialité dans le roman espagnol contemporain : bilan et perspectives

\title{
Autoréférence et « réalisme intentionnel » dans $L a$ Velocidad de la luz de Javier Cercas
}

et El vano ayer d'Isaac Rosa

\section{Anne-Laure Rebreyend}

\section{OpenEdition \\ Journals}

\section{Édition électronique}

URL : http://journals.openedition.org/bulletinhispanique/3534

DOI : 10.4000/bulletinhispanique.3534

ISBN : 979-10-300-0156-3

ISSN : $1775-3821$

Éditeur

Presses universitaires de Bordeaux

\section{Édition imprimée}

Date de publication : 1 décembre 2014

Pagination : 733-753

ISBN : 978-2-86781-963-6

ISSN : 0007-4640

Référence électronique

Anne-Laure Rebreyend, « Autoréférence et « réalisme intentionnel » dans La Velocidad de la luz de Javier Cercas », Bulletin hispanique [En ligne], 116-2 | 2014, mis en ligne le 01 décembre 2017, consulté le 04 mai 2019. URL : http://journals.openedition.org/bulletinhispanique/3534 ; DOI : 10.4000/ bulletinhispanique.3534 


\title{
Autoréférence et " réalisme intentionnel » dans La Velocidad de la luz de Javier Cercas, et El vano ayer d'Isaac Rosa
}

\author{
Anne-Laure Rebreyend \\ Université de Bordeaux Montaigne
}

Cet article propose une approche de la dialectique référencelautoréférence à partir de la théorie pragmatique du "réalisme intentionnel " de D. Villanueva. Puis il analyse la façon dont deux romans jouent, par des dispositifs relevant de l'autoréférence quoique de manière esthétiquement et éthiquement différente, sur les modes de référentialité réaliste du roman contemporain.

Mot-clés : «Réalisme intentionnel », réception, autoréférence, nouveaux réalismes.

Este articulo propone abordar la dialéctica referencialautorreferencia a partir de la teoría pragmática del "realismo intencional » de D. Villanueva. Luego analiza cómo dos novelas juegan, mediante dispositivos autoreferentes aunque de manera estética y éticamente diferente, con los modos de referencialidad realista de la novela contemporánea.

Palabras claves: « realismo intencional », recepción, autorreferencia, nuevos realismos.

This paper roots on a pragmatics of "intentional realism" by D. Villanueva to tackle the dialectics of referencelself-reference. The play on contemporary modes of realistic reference is analysed in two novels, both using self-referent tools although in different aesthetical and ethical perspectives.

Keywords: «intentional realism », reception, autoreference, new realisms.

$\mathrm{M}$ e saisir de la dialectique qui réunit ou oppose les concepts de référence et d'autoréférence pour l'étude de romans contemporains demande de préciser ses contours, notamment vis-à-vis des apports du colloque organisé 
par Geneviève Champeau en $1992^{1}$, dans le sillage duquel se situe cet ouvrage. Tout d'abord, si l'on entend par "référence " ce qui n'est pas le propre de la fiction, ce que la fiction ne peut pas accomplir du fait qu'elle a une dénotation nulle; et si, à l'inverse, on entend par " autoréférence " ce qui lui reste, ce qui est son mode de relation linguistique (la fiction ne renvoie qu'à elle-même), alors il semble difficile que ces deux outils servent véritablement à l'analyse de récits. Le débat paraît clos et la fiction condamnée à ne jamais parler du monde. Lidée de ne faire d'un antagonisme référence/autoréférence que l'outil de distinction entre récits factuels et fictionnels est un parti pris dont s'éloignent d'ailleurs nombre de communications du colloque de 1992. Juan Vila, par exemple, propose de la nuancer en considérant la distinction entre référence et autoréférence "en termes de degré de fictionalité " ${ }^{2}$, selon "l'axe graduel allant des productions les plus réalistes aux plus référentiellement abstraites » de Catherine Kerbrat-Orecchioni ${ }^{3}$. Ce qui m'intéresse particulièrement ici, c'est que le caractère réaliste d'un texte est assimilé à sa référentialité, définie comme un rapport linguistique au monde plus immédiat - quoique non transparent. $\mathrm{Si}$, comme l'ont généralement fait les participants au colloque de 1992, j'entends donc référence et autoréférence respectivement comme mouvement vers le monde (le texte parle du monde) et comme impulsion centripète (le texte pointe du doigt son élaboration), alors la référentialité serait l'apanage, voire la définition, du texte réaliste dans son acception la plus traditionnelle ou la plus intuitive.

Or, comme Geneviève Champeau en signalait la tendance en 1992 et comme l'évoque cet ouvrage, la littérature espagnole, depuis les années 1990-2000, est caractérisée par ce qu'on appelle un "retour du réel » ou " nouveau réalisme ", à partir d'une réappropriation de l'histoire récente. D'après un net consensus critique, elle ferait suite aux productions narratives " expérimentalistes " des années 1970, qui auraient renoncé à la référentialité pour se centrer sur une exploration de leur fonctionnement interne. La caractéristique de ces nouveaux récits, comme Jean-François Carcelen le suggère en $1992^{4}$, pourrait bien résider justement dans une combinaison paradoxale : la thématique du réel fait retour, mais associée à la métatextualité héritée du Nouveau Roman, renouvelant les réalismes historiques.

1. Geneviève Champeau (sous la dir. de), Référence et autoréférence dans le roman espagnol contemporain, Actes du colloque international de Talence (1992), Bordeaux, Maison des Pays Ibériques, 1994.

2. Juan Vila, «Les fantômes (auto)référentiels du ciné Roxy ", in G. Champeau (sous la dir. de), Référence et autoréférence..., op. cit., p. 81-94.

3. Catherine Kerbrat-Orecchioni, "Le texte littéraire : non-référence, auto-référence, ou référence fictionnelle? ", in "L'autoreprésentation. Le texte et ses miroirs ", Texte, Revue de critique et de théorie littéraire, $\mathrm{n}^{\circ} 1,1982$, p. 27-50.

4. Jean-François Carcelen, "Écriture de l'obsession et obsession de l'écriture : Cerbero son las sombras, de Juan José Millás ", in G. Champeau (sous la dir. de), Référence et autoréférence..., op. cit., p. 173-185. 
Comment la combinaison renouvelée de référentialité et d'autoréférentialité fonctionne-t-elle dans les récits contemporains ? Comment rendre l'épaisseur de sens de ces procédés ? Quel peut être le rôle de ces récits dans la société espagnole actuelle ? Je tenterai d'y répondre en deux temps. Le premier propose une approche de la dialectique référence/autoréférence à partir de la base théorique d'un modèle pragmatique du réalisme. Le second avance des pistes pour l'analyse de dispositifs d'écriture de deux ouvres: La velocidad de la luz, de Javier Cercas 5 , et El vano ayer, d'Isaac Rosa ${ }^{6}$. Pour cela, je partirai de quelques procédés d'autoréférence dans ces deux récits afin de montrer comment les textes jouent directement et très différemment sur les modes de référentialité réaliste du roman contemporain.

\section{L'ACTIVATION LECTORIALE DE LA RÉFÉRENCE, CONDITION D'opÉRATIVITÉ DE} LA FICTION DANS LE RÉEL

Ce premier temps théorique vise à poser un cadre d'analyse des textes et à démontrer comment la démarche pragmatique que développe Darío Villanueva dans Teorías del realismo literario ${ }^{7}$ permet de renouveler la réflexion autour de la dialectique référence/autoréférence. Celle-ci sera donc ici à la fois une catégorie d'analyse pour les textes et un objet de réflexion. Je voudrais contribuer à une approche du réalisme par la réception, comme y invite Geneviève Champeau. $\mathrm{Si}$, d'après Riffatterre, la référentialité est dans l'œil du lecteur', qu'est-ce qui la conditionne, dans chaque texte mais aussi dans tout ce qui constitue la formation préalable du lecteur?

Pour cerner l'opérativité de la notion de référence, la perspective de JeanMarie Schaeffer dans Pourquoi la fiction $?^{10}$ parait très pertinente. Le philosophe y réfléchit aux raisons anthropologiques pour lesquelles nous lisons des fictions, aux liens de ces dernières avec notre rapport au réel, à leur portée cognitive. Selon lui, ce serait passer à côté de la question que d'essayer d'y répondre en définissant des modalités de référentialité spécifiques à la fiction - spécifiques du fait que ses phrases ne renvoient pas à des référents réels. Ce type de définitions, qu'il appelle sémantiques, ne nous renseigne pas sur la portée cognitive des fictions. Il propose de renouveler :

5. Javier Cercas, La velocidad de la luz, Anagrama, 2005.

6. Isaac Rosa, El vano ayer, Seix Barral, 2004.

7. Darío Villanueva, Teorías del realismo literario, Madrid, Biblioteca Nueva, 2004. Toutes les citations, sauf mention spécifique, proviennent de sa première édition, Instituto de España/ Espasa Calpe, 1992.

8. Cf. par exemple Geneviève Champeau, "Recepción de la novela realista de posguerra ", in P. Aubert (sous la dir. de), La novela en España (siglos XIX y XX), Madrid, Casa de Velázquez, 2001, p. 207-219.

9. Michael Riffatterre, "L'illusion référentielle ", in R. Barthes et al., Littérature et réalité, Paris, Seuil, 1982 [1 $1^{\mathrm{e}}$ édition 1978], p. 93.

10. Jean-Marie Schaeffer, Pourquoi la fiction ? Paris, Seuil, 1999. 
la façon dont nous abordons en général le problème des relations entre la fiction et les autres modalités de la représentation (la perception, les croyances référentielles, la connaissance abstraite [...]), et donc la question de la manière dont les dispositifs fictionnels peuvent se rapporter à la réalité dans laquelle nous vivons. Nous avons tendance à vouloir réduire le premier problème à celui de la relation entre représentation fictionnelle et assertion référentielle; quant au deuxième, nous croyons que le nerf de la question réside dans la différence de statut entre les identités fictionnelles et les entités de la réalité physique. De ce fait, l'analyse de la fiction se trouve ramenée peu ou prou à celle du statut dénotationnel des propositions fictionnelles [...] et à celle du statut ontologique des entités fictives ${ }^{11}$.

Les limites des définitions sémantiques de la fiction proviendraient de l'assimilation de la fiction à l'énoncé verbal fictionnel : ces définitions " ne se sont jamais vraiment libérées du cadre dans lequel elles sont nées : celui d'une interrogation sur ce qui distingue les propositions portant sur des entités non existantes de celles ayant une référence ». Schaeffer substitue la question de la nature des relations que la fiction entretient avec la réalité par celle de comment elle opère dans nos vies ${ }^{12}$.

Car la fiction n'est pas qu'un ensemble de propositions : pour le philosophe, le premier critère qui la fonde est un cadre pragmatique, la "feintise ludique partagée " - la formule a connu une postérité certaine. Ce pacte repose à la fois sur une posture illocutoire de l'auteur, d'après Searle, et sur l'attitude modale adoptée par le lecteur qui, selon Schaeffer, transcende la question de la nature ou de la vérité des états de faits référés. D’une part, la fiction agit en modélisation mimétique; d'autre part, elle repose sur une attitude mentale, intentionnelle, spécifique. Par modélisation mimétique, il faut entendre qu'un modèle fictionnel est toujours une modélisation de l'univers réel, car, anthropologiquement, nous ne disposons pas d'une compétence de représentation qui serait spécifiquement réservée aux fictions :

Nous pouvons certes former des modèles posant des entités inexistantes, [...] inventer les univers les plus fantaisistes, mais [...] ces entités et ces univers seront des variantes conformes à ce que signifie pour nous " être une réalité ", parce que nos compétences représentationnelles sont toujours-déjà relatives à la réalité $[. .$.$] dans$ laquelle nous vivons ${ }^{13}$.

En revanche, l'activité de modélisation elle-même peut être un "commesi ", dans le cadre de fictions, c'est-à-dire que la fictivité d'un roman relève plus d'un critère illocutoire que de la fictivité de l'histoire elle-même ${ }^{14}$.

Ainsi la référentialité est-elle le seul mode de représentation, pour la fiction et la non-fiction. Outre la fictionalité ou non de l'énonciation, les critères qui déterminent la qualité de la réception de la fiction, la façon dont elle agit sur

11. Ibid., p. 200.

12. Ibid., p. 212.

13. Jean-Marie Schaeffer, Pourquoi la fiction?, op. cit., p. 218.

14. Cf. Barbara Herrnstein-Smith, On the margins of discourse, The University of Chicago Press, 1978, p. 29, citée par Gérard Genette, Fiction et Diction, Paris, Seuil, 2004 [1991], p. 156. 
nous, deviennent, selon Jean Alsina : les stratégies de persuasion narrative, comme un ethos de narrateur fiable, ou les réseaux de confirmation du sens par d'autres discours qui dotent de crédibilité les figures sémantiques du texte. À une dialectique du vrai et du faux qui opposerait récits de fiction et de non fiction se substitue la modalité unique de la croyance ${ }^{15}$. Pourtant, les récits hybrides contemporains, qui jouent sur les frontières entre les genres, les régimes factuel et fictionnel, en mimant notamment le discours de savoir historiographique, mettent en œuvre de complexes stratégies de véridiction. Nous verrons plus loin les problèmes que pose dans les romans un narrateur labile ou non fiable; ou encore comment des récits peuvent contrecarrer la possibilité pour le lecteur d'adhérer par confiance à tout discours reconnu. Face aux " fictions documentaires " ${ }^{16}$, il faut nuancer la traditionnelle assomption de l'irresponsabilité de la fiction, fondée sur l'idée que le texte fictionnel crée ses propres référents : si l'entrée dans un texte de fiction est censée court-circuiter la vérifiabilité du discours sérieux, comment se négocie cette «suspension de l'incrédulité » dans un récit hybride ?

Je voudrais à présent émettre l'hypothèse que c'est dans ce cadre théorique que fait sens le modèle du " réalisme intentionnel " de Darío Villanueva, qui approfondit l'analyse des modalités d'acceptation de la référence par le lecteur. Il y a un enjeu, dans le champ de la critique, à se saisir de sa démarche et à montrer ses liens fructueux avec d'autres théories, car, en dépit de critiques élogieuses, Teorías del realismo literario n'a pas rencontré beaucoup d'écho en France ni en Espagne. Rappelons les points forts de sa démonstration avant de discuter leur opérativité pour la production narrative contemporaine.

Darío Villanueva propose de renouveler la définition du réalisme par une démarche phénoménologique et pragmatique qu'il appelle le "realismo intencional». Elle me paraît particulièrement féconde pour étudier les écritures du réel actuelles, car elle propose de dépasser à la fois un réalisme qu'il nomme génétique, et qui prétend entretenir un rapport de correspondance avec la réalité, et un réalisme formel, fondé sur une autonomie totale du texte littéraire.

Para el primero, la realidad que precede a la obra encuentra su reflejo transparente en ella con la intervención de un arte literario que consiste [...] en el paradójico adelgazamiento de los medios que lo evidenciarían, sacrificados a aquel objetivo prioritario de recrear el referente exterior. Para el segundo, por el contrario, de la única realidad de la que se puede hablar es de la inherente y simultánea a la obra en sí, pues en ella nace y se constituye en ella ex novo ${ }^{17}$.

15. "Le référent c'est du dénoté, [...] de l'archivé, en un mot du vérifiable. Si, dans la conversation, garantie et vérification font partie de l'échange, dans la lecture littéraire, je me satisfais de la croyance en un vérifiable que je ne vérifie pas car je participe du simulacre ". Jean Alsina, "Référenciation, référentialisation - Los Bravos de Jesús Fernández Santos ", in G. Champeau (sous la dir. de), Référence et autoréférence ..., op. cit., p. 52.

16. Jean-François Carcelen, "Ficción documentada y ficción documental en la narrativa espańola actual: Ignacio Martínez de Pisón, Isaac Rosa », in G. Champeau et al. (sous la dir. de), Nuevos derroteros de la narrativa española actual: veinte años de creación, Universidad de Zaragoza, Prensas Universitarias de Zaragoza, 2011, p. 51-68.

17. Darío Villanueva, Teorías del realismo literario, op. cit., p. 69. 
Il réfute donc deux conceptions différentes du réel ainsi que du langage qui président respectivement au privilège de la référence - réalisme positiviste par correspondance avec le réel observé que le langage pourrait représenter parfaitement - et à celui de l'autoréférence - réalisme par cohérence interne au texte. Il les situe, au prix d'une simplification que le cours de son analyse vient nuancer, dans deux moments historiques distincts : le réalisme génétique correspondrait au XIX ${ }^{e}$ siècle; le second à l'école formaliste des années 1960$70^{18}$. Or, cette historicisation englobe tout le champ de l'institution littéraire, aussi bien les pratiques de création que les écoles théoriques et critiques, étroitement imbriquées. Il importe cependant de remarquer qu’à la nouvelle méthode d'analyse qu'il formule il n'associe pas de production littéraire qui lui serait contemporaine, contrairement aux réalismes génétique et formel. Cela suggère que sa méthode peut s'appliquer à tout type de textes. Toutefois, son évocation du Lazarillo et des Lettres de la religieuse portugaise puis de Truman Capote nous met sur une piste : les textes dont émanerait sa conception d'un "réalisme intentionnel " pourraient bien être spécifiquement ceux qui jouent de la frontière entre fiction et diction. De là à avancer que c'est l'étude des récits actuels exacerbant ce type de rapport complexe et paradoxal au réalisme, entre référence et autoréférence, qui tirerait le meilleur profit de sa démarche théorique, il n'y a qu'un pas, que je proposerai de franchir.

Sa méthode consiste à considérer le réalisme comme une pratique du lecteur, comme un mode de lecture. Adaptant la théorie communicationnelle de Grice au monde de la fiction, il postule "una asunción espontánea y natural por parte del lector de la seriedad de lo escrito en la obra, que aunque ficticio en su origen se prestaría a una descodificación realista " ${ }^{19}$. On retrouve la "suspension volontaire de l'incrédulité ". De façon spontanée, pour Villanueva comme chez Schaeffer pour qui la référentialité est le seul mode de représentation que l'on sait pratiquer, le lecteur projette sa propre expérience empirique de la réalité, son champ de référence ${ }^{20}$ (l'univers social et historique, mais aussi l'univers artistique et littéraire à travers les œuvres préexistantes $\left.{ }^{21}\right)$, sur celui du texte (le réseau de personnages, d'évènements etc., que le langage du texte

18. Ce faisant, il conjure leur possible retour dans la critique, notamment les excès du courant formaliste au cœur de sa formation universitaire, qui oblitérait la façon dont le lecteur fait parler le texte de sa propre réalité. Cf. Janet Paterson signalant avec humour comment la compénétration de la production littéraire et du discours critique configure la valeur artistique : "Ayant renoncé pendant la période du roman "représentatif " (où comme par mimesis la critique aussi ne s'intéressait qu’à la représentation) à identifier une pratique autoreprésentative, on démontre aujourd'hui [...] que le dernier signifié du texte, [...] le seul véritable, est celui où un roman nous parle de soi ». Janet Paterson, "L'Autoreprésentation : formes et discours ", in "L'autoreprésentation. Le texte et ses miroirs ", Texte, Revue de critique et de théorie littéraire, $\mathrm{n}^{\circ} 1,1982$, p. $177-195$.

19. Darío Villanueva, op. cit., p. 108.

20. Benjamin Harshaw, "Fictionality and Fields of Reference. Remarks on a theoretical framework ", Poetics Today, 5, 2, 1984, p. 227-251.

21. Ce n'est toutefois pas le monde à la réalité univoque et indubitable que postule le réalisme génétique, et qu'une épistémologie relativiste ou constructiviste est venue ébranler. 
institue dès sa première phrase en même temps qu'il s'y réfère). De là une nouvelle définition, phénoménologique et pragmatique : "el realismo literario es un fenómeno fundamentalmente pragmático, que resulta de la proyección de una visión del mundo externo que [...] cada lector aporta sobre un mundo [...] que el texto sugiere ${ }^{22}$. Si c'est la lecture réaliste qui active, actualise, le projet du texte, tout texte, même fantastique, peut être réaliste, du moment qu'il favorise l'immersion fictionnelle et la suspension de l'incrédulité, "pues detrás de ese complejo sistema de signos [...] hay siempre una referencia, actualizable e intencionable, bien a la realidad mostrenca y apariencial, bien a otra profunda, de esencias $»^{23}$.

Par cette nouvelle opérativité de la notion de référence, à laquelle il préfère le terme d'interprétant de Peirce et qui touche à la façon dont les lecteurs font parler au texte de leur réalité - " no del referente al texto sino de éste al lector y su contexto $»^{24}$ - se tisse un lien éloquent avec l'héritage de la théorie de la réception réapproprié par Ricœur ${ }^{25}$, et avec la théorie anthropologique et pragmatique de Schaeffer. L'ambition anthropologique commune à Villanueva et à Schaeffer est d'ailleurs significative d'un moment de reconfiguration de la place des études littéraires ; envisager la lecture en termes de compétence fictionnelle " naturelle », "spontanée " de l'homme s'inscrit dans la sphère d'influence des sciences cognitives - on verra plus loin le lien avec Monika Fludernik ${ }^{26}$ - qui ne se portent pas sur l'historicité des processus.

Villanueva apporte deux nuances, dont l'analyse qui va suivre fera jouer les clefs. D'une part, il déclare que le lecteur critique, par rapport au lecteur spontané, a tendance à court-circuiter son immersion, son actualisation réaliste des textes : "sólo un lector suspicaz, obsesiamente precavido contra las falacias intencional y afectiva, atento tan sólo al artificio artístico, hace una actualización deliberadamente antirrealista de la obra de arte literaria, ejecuta una "metalectura" ". À ce titre, le but que doit se fixer un critique à l'heure d'analyser un récit tiendrait à se dépouiller de ses artifices de défense contre la crédulité, pour accéder à sa réaction la plus spontanée au texte, afin d'opérer ensuite un retour critique sur les mécanismes déclenchés et trouver leur origine dans les procédés du texte.

Villanueva privilégie ce qu'il pourrait y avoir d'inné, d'ahistorique et d'agéographique dans ces processus. Pourtant, il concède une seconde

22. Darío Villanueva, op. cit., p. 119.

23. Ibid., p. 135.

24. Ibid., p. 158.

25. On retrouve ici la «mimesis III » de Ricœur, l'intersection du monde du texte en attente de lecture avec le monde du lecteur qui « refigure » la référence. Cf. Temps et récit 1, Paris, Seuil, 1983.

26. Sa définition du réalisme s'avère très proche de celle de Villanueva : "Realism in the sense in which I am using the term therefore closely corresponds to a mimetic representation of a human experience that cognitively and epistemically relies on a real-world knowledge. That realism is a significational effect, however: the result, that is, of the mimetic illusion that is transported into the reading process by readers' urge for narrativization ". Monika Fludernik, Towards a "natural" narratology, London/New York, Routledge, 1996, p. 38. 
nuance : la pratique du réalisme intentionnel est le fait d'une " communauté interprétative ${ }^{27}$ - ensemble de gens qui partagent les mêmes stratégies d'interprétation qui conditionnent, en l'actualisant, le sens des textes -, qui évoluent avec le contexte, les traditions, les institutions. Si « una época concreta puede favorecer la comunidad interpretativa del realismo intencional, y otra a la contraria $»^{28}$, l'école réaliste du XIX ${ }^{\mathrm{e}}$ siècle a, par exemple, tout particulièrement modelé la pratique mimétique réaliste : "estamos dispuestos a admitir que en el siglo XIX el realismo no sólo creó una escuela de escritura, sino que perfeccionó sobremanera la escuela -o comunidad interpretativa- de los lectores realistas intencionales $»^{29}$, concède Villanueva. L'évolution des horizons d'attente du lectorat dépend des discours concurrents à la littérature ou par rapport auxquels elle se place - aujourd'hui, les sciences humaines et sociales ou le journalisme notamment, mais aussi toutes les politiques de mémoire en Espagne.

C'est dans cette perspective, celle de l'historicité du fait littéraire, que Judith Lyon-Caen propose de saisir dans le temps le rapport à la référentialité des textes réalistes, comme objet de recherche historiographique ${ }^{30}$. En d'autres termes, les catégories de référence et d'autoréférence seraient des outils d'analyse à exploiter, mais aussi à historiciser, avec leurs usages. Ainsi, pour Antoine Lilti et Etienne Anheim, une des meilleures manières d'éviter d'opposer la fiction aux discours référentiels ou de conclure à la libre irresponsabilité de la fiction autoréférente

est peut-être de reposer [la question] du réalisme, non pas dans le sens restreint que lui a donné l'histoire littéraire, mais dans le sens plus large de la vocation référentielle de la littérature, de sa tentative de décrire et de donner sens au monde. [...] Dans cette optique, il s'agit moins de s'interroger sur les genres traditionnels de l'histoire littéraire que sur ceux qu'une histoire des capacités cognitives de la littérature met en lumière ${ }^{31}$.

Comment appliquer à présent cette façon renouvelée de voir le champ du littéraire, du réalisme et de la lecture - modèle qui emprunte à la phénoménologie, à l'esthétique de la réception, à la pragmatique -, à l'analyse de textes ? À défaut de développer ici une étude empirique de la réception, comme y invite Villanueva et comme l'a fait Judith Lyon-Caen sur les usages des lecteurs réalistes au XIXe siècle, je voudrais essayer de retrouver quelques unes de ces pistes dans deux romans.

27. Stanley Fish, "Interpreting the Variorum », Critical Inquiry, 2, 3, 1976, p. 465-485, cité par Darío Villanueva, op. cit., p. 148-149.

28. Darío Villanueva, op. cit., p. 149.

29. Ibid., p. 150. Cf. Isabel Román Román, «El juego con las expectativas del lector realista: la narrativa de la primera vanguardia española » in G. Fabry et C. Canaparo (sous la dir. de), El enigma de lo real. Las fronteras del realismo en la narrativa del siglo XX, Oxford, Peter Lang, 2007. L'indistinction qu'elle opère p. 58-59 entre lecteur traditionnel de roman et lecteur de romans du $\mathrm{XIX}^{\mathrm{e}}$ siècle confirme que les canons du XIX ${ }^{\mathrm{e}}$ siècle ont durablement modelé le mode de lecture.

30. Judith Lyon-Caen, La lecture et la vie, Taillandier, 2006 ; Judith Lyon-Caen et Dinah Ribart, L'historien et la littérature, Paris, La Découverte, 2010.

31. Antoine Lilti et Etienne Anheim, "Introduction ", in "Savoirs de la littérature ", Annales. Histoire, Sciences sociales, n 2, mars-avril 2010. 
2. Configuration d'un lecteur implicite « RÉAliste » DANS LA VELOCIDAD DE LA LUZ ET EL VANO AYER

Darío Villanueva invite à contribuer à un répertoire de formes "réalistes " en changeant le point de vue qui y préside traditionnellement ${ }^{32}$. Il reprend à son compte ceux, relativement traditionnels, que relève Philippe Hamon ${ }^{33}$, mais qu'il explique par une approche pragmatique et non plus génétique : non pas en tant qu'ils connectent au mieux avec la réalité mais en tant qu'ils engendrent chez les récepteurs empiriques une coopération réaliste. Mon ébauche d'approche par les romans El vano ayer et La velocidad de la luz, que j'aborderai successivement et que j'ai choisis pour leurs options esthétiques assez diamétralement opposées, vise plusieurs objectifs. D'une part, je m'empare de quelques-uns des outils rassemblés par Villanueva pour les tester à la lumière des récits hybrides : la configuration d'un lecteur implicite représenté dans le texte, ainsi que deux arguments d'authentification que sont la construction d'une figure de narrateur fiable ${ }^{34}$ mais aussi d'un auteur implicite par effet de cohérence de la structure. Cela déploie de nouveaux pans de cet éventail de procédés réinvesti par Villanueva, en donnant toute leur force persuasive aux dispositifs d'autoréférence qui pourtant, selon Villanueva et avec lui la grande majorité des théoriciens, ne sont pas propices au réalisme puisqu'ils attirent l'attention sur l'artefact littéraire. Il s'agit notamment de rendre à deux procédés associés, l'autofiction et la métatextualité (l'insertion d'un personnageécrivain et les commentaires sur l'écriture en train de se faire), leur épaisseur, leurs paradoxes, dans le cadre de la "suspension de l'incrédulité " qu'est censé adopter un lecteur de fiction. D'autre part, je propose d'essayer de considérer la portée éthique de ces textes à partir de la façon dont les deux récits gèrent les " communautés d'interprétation " que constituent les lecteurs.

El vano ayer est un roman de la mémoire qui porte sur la répression policière à la fin du franquisme et sur les liens de continuité du régime dictatorial jusque dans le présent via la transition démocratique. Dans le même temps, l'instance narratrice, en proie avec l'écriture de son livre, dénonce les écueils de la fiction mémorielle des années 1990-2000 (télévisuelle, cinématographique, romanesque), tant du point de vue des choix thématiques que des procédés formels. Le récit montre comment l'univers de référence du lecteur est conditionné par un contexte de production culturelle dominante. En d'autres termes, El vano ayer analyse la communauté interprétative de la fiction de

32. Darío Villanueva, op. cit., p. 166.

33. Philippe Hamon, "Un discours contraint ", in R. Barthes et al., Littérature et réalité, op. cit., [1 $1^{\mathrm{e}}$ édition 1973], p. 119-181.

34. "otro de los principios más evidentes para el logro de un discurso realista es su fundamentación en una fuente de origen dotada de autoridad fidedigna que se granjee la confianza del lector empírico ", Darío Villanueva, op. cit., p. 176. Voir aussi Wayne Booth, "Distance et point de vue", in R. Barthes et al. Poétique du récit, Paris, Seuil, 1977, p. 101 : analyser la distance entre auteur, narrateur et lecteur " consiste à s'interroger sur l'effort déployé par l'auteur pour maintenir ou détruire l' "illusion réaliste" ". 
la mémoire en Espagne. La critique générique à laquelle le roman se livre procède par discours métanarratif et autres procédés qui exhibent, notamment par la parodie, le pastiche ainsi que la métaphore du spectacle ${ }^{35}$, les schémas narratifs topiques des fictions de la mémoire. D'après la règle généralement admise de l'effacement du langage pour figurer une transparence référentielle et favoriser l'immersion fictionnelle, ces dispositifs autoréférents devraient se situer aux antipodes d'un répertoire de procédés réalistes, y compris dans le sens villanuevien du réalisme. Pourtant, la natural narratology de Monika Fludernik, dont l'intérêt pour les comportements cognitifs "spontanés » qui président à l'interprétation des textes n'est pas sans faire écho au " réalisme intentionnel ", nous donne une clef théorique pour comprendre comment ces dispositifs peuvent opérer comme un méta-réalisme, en dépit du fait qu’ils brisent par certains aspects la suspension de l'incrédulité :

Experimental fiction can be read as intertextual play with language and with generic modes, and this -since it projects an intentional meta-narrative function-is a mimetic strategy just like any other. Reading experience in terms of making sense of the text can be conceptualized as a deliberately anti-narrative discourse which is then recuperated as the product of rhetorical strategy on the part of the author. Such experimental texts are therefore not mimetic in terms of reproducing, if in a different medium, a prototypical version of narrative experience, but are mimetic in their structured anticipation of reader's attempts at reinterpreting them mimetically if only at a meta-meta-realist level of a self-reflexive, explicitly anti-mimetic language game. [...] that sense-making, since it always relies on natural categories of cognition, is by definition mimetic. Experimental fiction is in fact read against its grain since readers, in so far as they read such texts as narrative, supply the inexplicit narrativity of the text by extrapolation from its anti-narrative surface ${ }^{36}$.

La proposition de Fludernik part d'une définition spécifique de la narrativité comme " mediated human experienciality ${ }^{37}$ : est narratif, ou mimétique, ce qui représente une expérience humaine et me la fait éprouver. En fonction de cette définition, un discours autoréférentiel sous sa modalité métatextuelle (discours qui parle de sa propre élaboration) et intertextuelle (qui signale le réseau des textes dans lequel il se place) ne me plonge pas dans la conscience d'un personnage et dans son expérience du monde (" a prototypical version of narrative experience "). En revanche, il s'avère mimétique au second degré et à deux niveaux. D'une part, l'expérience humaine qu'il représente, par rapport à un réalisme traditionnel, est celle de la lecture. La fiction expérimentale anticipe et modélise l'interprétation lectoriale (" structured anticipation of reader's attempts at reinterpreting them mimetically") ; c'est en ce sens qu'elle serait mimétique au second degré. D'autre part, elle produit bien chez le lecteur l'opération mimétique d'interprétation, de décodage, qui consiste en une dynamique cognitive de projection de mon monde de référence sur le

35. Anne-Laure Rebreyend, "Deconstrucción del realismo. Paradojas de la metáfora del teatro en El vano ayer, de Isaac Rosa ", Pasavento, Revista de Estudios Hispánicos, vol. II, $\mathrm{n}^{\circ} 1$, invierno 2014, p. 57-76.

36. Monika Fludernik, op. cit., p. 36.

37. Ibid. 
monde du texte ("that sense-making, since it always relies on natural categories of cognition, is by definition mimetic "). La définition de la mimesis par Fludernik concorde donc avec celle Villanueva en ce qu'elle fait du réalisme une démarche cognitive du lecteur par laquelle il apporte du sens au texte : «mimesis must NOT be identified as imitation but needs to be treated as the artificial and illusionary projection of a semiotic structure which readers recuperate in terms of a fictional reality $"{ }^{38}$. Ainsi un texte métanarratif, quoiqu'il brise les codes de l'immersion fictionnelle, porte-t-il finalement au second degré le projet de tout récit. Ce cadre permet d'expliquer le méta-réalisme autoréférentiel réflexif de El vano ayer, qui tient, en outre, à ce qu'il représente, plus que le réel, les discours sur le réel qui construisent notre rapport au monde.

El vano ayer déconstruit les modes réalistes traditionnels (celui de Galdós mais aussi du réalisme des années 1950) et contemporains (le roman de la mémoire) en signalant leur patron commun hérité du mélodrame. Il fustige, entre autres, deux de ses caractéristiques : le recours au pathos et la trame verrouillée par une structure chronologique et causaliste qui ne laisse rien au hasard. Pourtant, une analyse plus poussée du roman démontrerait qu'il sacrifie au même effet de cohérence structurelle. En effet, d'une part, la construction du récit est faite de parallèles et d'échos dont le réseau ne laisse pas le sens si ouvert qu'il y paraît. Ce réseau fonctionne par récurrence d'expressions (le discours franquiste sur la société et la répression ${ }^{39}$ ), par retours de personnages (Julio Denis, «Guillermo Birón ») qui finissent par reconstituer des trajectoires en dépit de la structure fragmentaire, ou encore par insistance sur des thèmes-leitmotive qui connaissent plusieurs traitements au cours du récit (la torture, le traitement fautif du passé par la fiction...). D'autre part, de multiples suggestions provenant soit de l'instance narrative omniprésente, soit des diverses voix qui se trouvent ponctuellement en charge du récit, incitent à combler les vides de l'intrigue et les questions sans réponses ${ }^{40}$. Certes, en même temps qu'elle nous les fournit, l'instance narrative ironise sur les éléments qui invitent à forcer l'apparente indécision du sens. Les plus longs développements narratifs qui construisent le parcours des personnages et l'avancée de l'intrigue sont autant de concessions à la narrativité offertes au lecteur avec une ironie mordante. Lorsque le narrateur annonce une hypothèse plus ferme sur un événement, une interprétation catégorique, un chapitre « romanesque » sur un personnage, son discours est miné par les signes de sa non-fiabilité. L'encadrement du chapitre mettant en scène une sombre soirée de Julio Denis dans les faubourgs (p. 221 à 242) en est un bon exemple : le chapitre précédent prend soin d'annoncer qu'il s'agira d'un développement fictionnel mélodramatique ("estamos construyendo una ficción, señoras y señores,

38. Ibid., p. 35.

39. Cf. Anne-Laure Rebreyend, op. cit., p. 61-65.

40. "A veces es necesario abandonar por un momento ambigüedades», p. 155 ; "Para desactivar definitivamente las sospechas sobre Julio Denis [...] proponemos un posible relato biográfico del profesor", p. 172 ; "va siendo hora de espantar las últimas dudas, esas esperanzas que algunos mantienen de una vuelta de tuerca final en la intriga", p. 242 ; " el azar sólo es un modo de la causalidad cuyas reglas ignoramos", p. 277. 
relájense y disfruten ", p. 220), tandis que le suivant interpelle brutalement le lecteur dont il rompt l'immersion ("Mucho mejor asi, ¿no es cierto? Siempre será más aceptable esta comedia de arrojos seniles", p. 242). Aussi faut-il comprendre par antiphrase les prétendues concessions à la narrativité qu'elle prétend offrir au lecteur avide d'indices et de repères narratifs. Néanmoins - il faudrait vérifier si ma propre expérience est représentative par une étude empirique auprès de lecteurs -, les conjectures proposées en guise de trajectoire des personnages sont bien les seuls éléments qui font avancer l'intrigue. Lorsque le narrateur propose une option narrative, même en la disqualifiant, elle fait partie du récit, elle devient performativement le récit. Le lecteur se raccroche aux pastiches mélodramatiques qui émaillent le texte comme à des balises narratives au sein d'un récit fragmentaire construit par le discours métanarratif superlatif. Cela viendrait confirmer la force de la «spontanéité » de l'immersion fictionnelle qui est, d'après Villanueva, la condition du réalisme ; et ce, y compris au sein d'une fiction qui déconstruit et semble vouloir court-circuiter ce type de réalisme. C'est précisément ce qu'affirme Fludernik : "Experimental fiction is in fact read against its grain since readers, in so far as they read such texts as narrative, supply the inexplicit narrativity of the text by extrapolation from its anti-narrative surface ${ }^{41}$. El vano ayer procède en activant simultanément ou alternativement ces deux modes : esthétique et visée anti-narrative (" anti-narrative surface ») et éléments d'une narrativité explicite qui donnent au lecteur du grain à moudre pour son immersion réaliste (l'« extrapolation" mimétique dont parle Fludernik).

Selon Philippe Hamon, "l'effet de réel n'est que la conséquence de la perception d'un effet de structure, réinterprété lui même comme effet d'autorité. La croyance en l'existence des faits relatés passe non pas par la connaissance de ces faits, mais par le crédit que le lecteur attache à tout structurateur de faits, l'auteur absent qu'il déduit de la structure même du texte " ${ }^{42}$. L'effet d'autorité qui favorise la lecture réaliste naïve, ou mélodramatique, du texte, en dépit des mises en garde de l'instance narrative, fonctionne donc par le biais de la structure du texte, nous venons de le voir, mais aussi par la configuration de l'auteur implicite, qui lui est liée. Il y a deux niveaux d'auteur implicite, dans sa définition théorique et dans ce texte : la figure de l'auteur qui apparaît effectivement représentée dans le texte, et celle que seul le lecteur projette à partir de son interprétation ${ }^{43}$. Celle qui apparaît représentée dans le texte, c'est ce que nous avons appelé « instance de narration " ${ }^{44}$. En revanche, la figure qui

41. Monika Fludernik, op. cit., p. 36.

42. Philippe Hamon, "Thème et effet de réel », op. cit., cité par Villanueva, op. cit., [édition de 2004], p. 186.

43. Cf. Tom Kindt, "L' "auteur implicite”. Remarques à propos de l'évolution de la critique d'une notion entre narratologie et théorie de l'interprétation ", in J. Pier (sous la dir. de), Théorie du récit. L'apport de la recherche allemande, Presses universitaires du Septentrion, 2007.

44. Certains courants théoriques remettent en question, au contact de textes auxquels me semblent correspondre ceux qui nous occupent, la distinction entre narrateur et auteur. La catégorie d' "auteur implicite " a fait partie des premières étapes qui sont venues nuancer cette distinction ; c'est en partie l'équivalent du terme d' "instance de narration " proposé par 
est le fruit de notre interprétation se modifie en fonction de notre approche critique. La brève analyse que je viens de mener nous engage à prendre de la distance par rapport aux suggestions de l'auteur implicite représenté. C'est d'autant plus clair lorsqu'il exhibe par de très nombreuses expressions sa toutepuissante maitrise du cours du récit, scellant ainsi l' " effet d'autorité " que Villanueva investit dans son modèle. À ce titre, un chapitre très significatif (p. 182-188) est émaillé de verbes qui dénotent la puissance et la coercition sur les péripéties des personnages : "podemos forzar la situación", "podemos obligar a uno de los estudiantes a olvidar", "no le permitiremos mirar atrás", "podemos decidir que sea herido", etc. L'effet d'autorité provient donc de deux niveaux. Au premier degré, il tient à la démonstration de force d'un narrateur - ici l'équivalent de l'auteur implicite représenté - à la merci duquel se trouvent le récit et le lecteur. Au second degré, on peut conclure que par différents procédés, l'auteur implicite non représenté, à savoir celui que notre analyse nous conduit à deviner, nous incite à nous méfier du précédent, qui apparaît non fiable car mimant des pratiques autoritaristes des narrateurs du réalisme traditionnel.

Enfin, à ces deux figures d'autorité correspondent très exactement deux figures de lecteur implicite représentés dans le texte, interpelés même par l'instance de narration ou auteur implicite représenté. Dans la perspective du "réalisme intentionnel ", il s'agit d'un personnage-témoin qui nous indique le type de lecture et d'interprétation à adopter ${ }^{45}$. Cette instance est essentielle, selon Villanueva, à la coopération réaliste des lecteurs empiriques, c'est à dire nous. Dans El vano ayer, on en trouve deux modèles antagoniques : le lecteur qui collabore aux procédés du réalisme mélodramatique vilipendé, contre le lecteur critique qui leur résiste. Le premier, qualifié tantôt ironiquement de "sabio público lector» (p. 15), tantôt vertement de "lector perezoso » (p. 21), par quoi il faut entendre "lector hambrient [o] de intriga» (p. 172), est présenté comme le modèle majoritaire, cible du commerce éditorial, passif dans le rapport de force imposé par le narrateur et idéologiquement conservateur (p. 81). Le second est caractérisé par sa lucidité face aux techniques narratives ("los lectores menos crédulos ", p. 18), son intransigeance (" el lector menos complaciente», p. 18), et son positionnement politique critique face à la Transition démocratique ("un grupo de radicalizados lectores", p. 189, "una minoría revoltosa ", p. 192).

Ainsi, El vano ayer énonce un refus des chemins que Villanueva dirait " innés » ou "spontanés » du réalisme intentionnel, que l'instance de narration identifie comme hérités du mélodrame et dont elle traque les modalités. Le récit procède en ciselant une dialectique complexe de frustration-satisfaction des attentes du lecteur. Il met en scène deux lecteurs implicites antagoniques,

plusieurs hispanistes pour analyser les récits contemporains. À ce débat sur narrateur/auteur implicite/auteur correspond en miroir une hésitation entre les termes de narrataire/lecteur implicite/lecteur.

45. Darío Villanueva, op. cit., p. 172-174. Il s'agit de "generar en los receptores empiricos [...] un efecto de reconocimiento intencional de su propia realidad ". Ibid., p. 164. 
qui pourraient incarner l'oscillation entre adhésion naïve et esprit critique au sein de chaque lecteur. C'est sa version la plus lucide et exigeante qu'appelle de ses vœux l'auteur implicite du roman ; ce faisant, il l'active en chaque lecteur, au prix d'une incitation au refoulement du plaisir d'immersion. Une formation à la lecture dont l'implication politique est à la source de l'engagement d'Isaac Rosa : à lecteur critique, citoyen actif.

D'une certaine façon, La velocidad de la luz, de Javier Cercas, pratique le type d'écriture que moque et fustige Elvano ayer ${ }^{46}$. Sa publication suit immédiatement celle de Soldados de Salamina ${ }^{47}$ dans la bibliographie de Cercas, et les deux romans comportent de nombreuses similitudes. C'est d'autant plus important que Soldados de Salamina occupe une place clef dans la littérature espagnole actuelle. Il est considéré par les critiques, du fait surtout de son spectaculaire succès de publication et de sa postérité, comme l'un des détonateurs de la fiction mémorielle en Espagne. Il aurait joué, à ce titre, un rôle significatif dans la reconnaissance d'un savoir propre à la littérature, pour répondre aux attentes du public face à des institutions qui ont échoué à proposer un récit collectif juste et réparateur de la Guerre Civile et du franquisme. On touche sans doute ici à l'une des raisons profondes d'un retour de la référentialité réaliste en Espagne, une modalité de l'historicité du pouvoir cognitif attribué à la littérature. Pour toutes ces raisons, qui reviennent à dire que la communauté interprétative des romans de la mémoire en Espagne est nourrie du paradigme fictionnel fondé en partie par Soldados de Salamina, il me semble judicieux de lire La velocidad de la luz en lien avec le roman de Cercas qui le précède ; je crois l'inverse vrai également.

En effet, on retrouve dans les deux romans une structure en miroir entre un témoin soumis au traumatisme d'une guerre - ici Rodney, un vétéran de la guerre du Vietnam - et un narrateur-personnage qui aspire à écrire un roman. Les deux récits s'articulent autour du déroulement d'une enquête, centrée sur la référence au passé, progressivement éclipsée par la mise en scène autoréférente du processus de l'écriture en marche, alimentée par le déploiement de l'autofiction caractéristique de l'univers fictionnel de Cercas. On peut parfaitement dire de La velocidad de la luz, comme Georges Tyras le dit de Soldados de Salamina, que l'histoire de Cercas aux prises avec l'écriture de son roman s'impose alors comme l'enjeu central de la narration ${ }^{48}$. Cette supplantation de la référence par l'autoréférence est au cœur des critiques éthiques formulées à l'encontre de Soldados de Salamina.

Pour essayer d'apporter un éclairage différent en analysant les éventuelles modalités et implications d'un "leurre " similaire ${ }^{49}$ dans La velocidad de la

46. El vano ayer mentionne d'ailleurs explicitement (p. 18-19), dans son catalogue de topiques, le paradigme d'écriture popularisé par Soldados de Salamina de Cercas : le « relato real ».

47. Javier Cercas, Soldados de Salamina, Barcelona, Tusquets, coll. Andanzas, 2001.

48. Georges Tyras, "Rafael Sánchez Mazas ou la sale mine du récit réel (sur Les Soldats de Salamine, de Javier Cercas) ", Témoigner. Entre Histoire et Mémoire, Revue pluridisciplinaire de la fondation Auschwitz, éditions Kimé, n 102, janvier-mars 2009, p. 66.

49. Jean-Marie Schaeffer, Pourquoi la fiction? op. cit., p. 199 : «la fiction procède certes à 
$l u z$, je procèderai, comme le réclame Villanueva, par dédoublement entre mon expérience d'immersion fictionnelle et mon analyse critique postérieure, afin de discerner les procédés qui m’ont incitée à procéder à une lecture réaliste. Généraliser mon expérience de lecture singulière en norme lectorale comporte des limites évidentes ; je tenterai néanmoins de prouver qu'examiner le jeu d'allers et retours entre adhésion naïve et lecture critique face au déroulement du récit nous ouvre de nouvelles portes d'interprétation et d'évaluation de ce roman. Comme nombre de lecteurs, j'ai fait l'expérience d'une intense immersion dans le roman. Mon hypothèse est que cette adhésion première passe par un effet d'autorité et de cohérence de la structure, deux procédés stimulant le "réalisme intentionnel ", comme nous l'avons vu ; tandis que s'opère dans un deuxième temps une prise de recul par rapport au narrateur, portée une nouvelle fois par une figure de lecteur implicite, qui invite à un jugement éthique nuancé sur le propos du récit.

Examinons quelques dispositifs qui successivement nouent la confiance en la vérité du récit, puis incitent à la suspension de la crédulité du lecteur. Les premiers tiennent à des stratégies de séduction et de persuasion autour de la figure du narrateur, un pseudo-pacte autobiographique, la structure non problématique du roman, son jeu d'enquête et de dévoilement progressif ainsi que la mise en parallèle entre Rodney et le narrateur, qui permet une lecture relativement aisée. Les indices qui fondent le pacte autofictionnel (éléments biographiques, référence aux ouvrages précédents de Cercas) sont de puissants facteurs d'adhésion à la vérité du récit, tout comme la démarche d'enquête autour du personnage de Rodney. Le régime de vérité est également configuré par une éventuelle connaissance préalable du pacte de Soldados de Salamina par le lecteur : la mimesis de méthode historiographique qui y est à l'œuvre fait présumer, consciemment ou non, d'un travail de documentation historique rigoureux dans La velocidad de la luz et donc de sa référentialité d'ordre factuel. Par plaisir, je suspends mon incrédulité et investis d'une valeur de véridicité les indices de référence au monde réel. C'est la force de l'autofiction et du pacte fiction/factualité qu'adoptent nombre de récits contemporains. L'ethos du narrateur parachève le tout : il semble entreprendre une sorte de trajectoire de maturité, conjurant l'auto-complaisance, marquée par la volonté d'élucider les failles existentielles de son ami Rodney ainsi que de donner du sens à sa propre vie. Le contrat de sincérité semble aussi assuré par la multiplication des modalisateurs visant à convaincre de sa prudence de jugement, comme lorsqu'il affiche massivement la défaillance de son souvenir ${ }^{50}$, ou encore par une série de paralipses ${ }^{51}$, restrictions de la focalisation à l'information passée et partielle

travers des leurres [...], mais son but n'est pas de nous leurrer, d'élaborer des illusions : les leurres [...] sont simplement le vecteur grâce auquel elle peut atteindre sa finalité véritable ».

50. "no recuerdo por qué lo hice", "no recuerdo lo que nos dijimos" (p. 202), " mi recuerdo de las horas que siguieron es aún más inseguro" (p. 206)...

51. "Les deux types d'altérations concevables [aux règles de la focalisation d'un récit, ici un récit rétrospectif à la première personne] consistent soit à donner moins d'information qu'il n'est en principe nécessaire, soit à en donner plus qu'il n'est en principe autorisé dans le code 
du héros en dépit de ses connaissances postérieures : le narrateur prouve ainsi qu'il cherche à restituer, sans la déformer, sa perception passée. La présence d'un personnage-écrivain qui communique un certain nombre d'hésitations d'écriture et nous livre son histoire en marche n'apparaît pas comme un facteur de perturbation de la lecture réaliste intentionnelle : au contraire, elle scelle un pacte complexe qui prendrait acte des imperfections de son narrateur pour poursuivre une sorte de contrat de sincérité.

En contraste, toutefois, tous ces signes sont oblitérés par le contrôle total de la narration que le narrateur déploie, créant cet effet d'autorité abordé plus haut. On note, tout au long du récit, des incursions dans l'esprit du protagoniste extrêmement précises, à la limite du vraisemblable, qui reviennent de façon presque superflue sur les errements de la pensée du héros. En voici un exemple :

Inmóvil, recuerdo que pensé: tiene tantas ganas de reir que no puede reir. Luego pensé: no, está llorando y seguirá llorando y no va a dejar de llorar, si es que alguna vez deja de llorar [...]. Luego pensé: no, tiene tantas ganas de llorar que no puede llorar. Luego pensé: no, tiene miedo, un miedo afilado como una hoja de afeitar, un miedo que corta y sangra y hiede y que yo no puedo entender. Luego pensé: no, está [...] tan loco que es capaz de engañarnos y fingir que está cuerdo. (p. 50-51) ${ }^{52}$

Paradoxe significatif que cet écart entre les incertitudes mémorielles que nous avons mentionnées et ces démonstrations de mémoire omnipotente, capable d'épouser les doutes et interprétations divergentes qui traversent le protagoniste en quelques secondes, alors que de nombreuses années séparent le temps de l'histoire de celui du récit. Ces paralepses, incursions trop précises dans la conscience du personnage focal pour être vraisemblables, frisent ici l'omniscience et rompent l'illusion de factualité avec laquelle flirte le récit.

On comprend progressivement que le narrateur manipule le lecteur, formule des promesses qu'il ne tient pas, finit par se placer au cœur d'un récit qu'il prétend destiné à la transmission de l'histoire d'un autre. La distance morale et temporelle qui sépare au début du récit le narrateur d'âge mûr et son plus jeune moi finit par disparaître. On note une multiplication des formules mélodramatiques comme "entonces entendi de repente lo que no habia entendido aquella noche [...] supe que era el hombre más sólo del mundo [...] y entendí también que [...] las tornas habian cambiado" (p. 219), "Ocurrió hace tres años, pero no ocurrió por azar" (p. 153) : jouant d'un suspense cliché que le narrateur prétendait éviter au début du roman, il affiche une sorte de nécessité des péripéties, de cohérence déterministe au sein de l'histoire dont il montre qu'il tient fermement, voire tyranniquement, les rênes.

de focalisation qui régit l'ensemble. Le premier type porte un nom en rhétorique [...] : il s'agit de l'omission latérale ou paralipse. Le second ne porte par encore de nom ; nous le baptiserons paralepse [...] » Gérard Genette, Discours du récit, Paris, Seuil, 2007 [1972], p. 201. Le choix, par un narrateur autobiographique, de se limiter à l'information passée du héros est une modalité de paralipse.

52. On trouve un autre exemple p. 35 : «Ya digo: primero pensé que bromeaba; luego pensé que no se refería a lo que yo creía que se refería y le miré a los ojos y pensé que no bromeaba; luego volvi a pensar que bromeaba y luego ya no supe que pensan». 
Pris dans les filets persuasifs du roman et notamment de l'ethos de narrateur fiable campé au début du récit, le lecteur aura pourtant sans doute tôt fait d'oublier ces indices, qui relèvent des modalités structurelles et langagières de la narration, destinés à provoquer une réaction de rejet à l'égard du narrateurpersonnage. Contrairement à $E l$ vano ayer qui intègre et exhibe le discours métatextuel critique tout au long du roman, il faut attendre véritablement la fin de La velocidad de la luz pour que le lecteur soit forcé à un retour critique. C'est à partir de la fin de la troisième partie de l'ouvrage, qui porte sur l'ivresse de succès littéraire et médiatique éprouvée par le personnage-narrateur, que son histoire donne les clefs morales qui établissent sa non fiabilité : il s'avère infidèle, outre son entreprise narrative comme on l'a vu, à ses amis, à sa femme qu'il néglige et humilie, ou à sa vocation d'écrivain ("le perdi el respeto a la literatura ", p. 201). Il paraît essentiel d'interpréter l'ensemble de ces éléments comme facteurs d'émergence d'un auteur implicite dont le système de valeurs se distingue de celui du narrateur, envers lequel le lecteur est voué à formuler un jugement éthique négatif, selon les termes de James Phelan ${ }^{53}$.

Il s'avère que le procédé qui mime le plus clairement cette démarche est l'apparition d'une figure de lecteur implicite représenté dans le texte. On a vu que El vano ayer procédait également ainsi, mais le sens en est nettement différent du fait de la chronologie de l'expérience de lecture, car ce personnage-témoin de lecteur n'apparaît qu'à la fin de La velocidad de la luz - et de manière assez cryptée. Il s'agit du personnage de Jenny, la femme de Rodney. À travers une correspondance suivie, le personnage-narrateur tente de la persuader de vivre avec lui afin de sceller son propre fantasme de remplacer Rodney en adoptant sa famille. Sa correspondance vise à convaincre subrepticement Jenny de l'évidence de leur union, tout comme il a construit son récit de manière à persuader le lecteur de son innocence à l'égard du crime dont il se sent coupable. Pourtant, Jenny ne collabore pas à cette manipulation et, tout en ayant pris plaisir à leurs échanges, rétorque : "Esto no es álgebra ni geometria: cuando se trata de personas dos más dos nunca suman cuatro. Quiero decir que nadie puede sustituir a nadie" (p. 293-294). Ce qu'elle récuse ainsi n'est rien moins que la colonne vertébrale de la construction du récit, à savoir la mise en parallèle existentielle entre Rodney et le narrateur-personnage. Ainsi Jenny se constitue-t-elle en paradigme du bon lecteur, entre immersion naïve, plaisir de lecture, et recul critique sur la personne du narrateur.

Dernière étape de cet itinéraire de lecture et de l'épiphanie : comme dans Soldados de Salamina ${ }^{54}$, les véritables héros du roman sont les personnages secondaires, en dépit de la place étouffante que prend le narrateur dans son récit. En effet, il appartient au lecteur attentif de percevoir la norme de l'auteur implicite par l'exemplarité des valeurs de ces trois personnages, à l'opposé

53. James Phelan, Experiencing fiction: judgments, progression and the rhetorical theory of narrative, Ohio State University Press, 2007.

54. Georges Tyras abonde dans le sens de Jean-François Carcelen, cf. «Rafael Sánchez Mazas ou la sale mine du récit réel... ", op. cit., p. 63. 
des milieux médiatique et universitaire dans lesquels évolue le personnagenarrateur, dépeints comme superficiels et égoïstes. Les actes de Rodney, de son épouse Jenny et de Marcos, le meilleur ami du narrateur, sont placés sous le signe du courage, du dévouement et de la fidélitée ${ }^{5}$; de façon très significative pour le sens du récit, c'est Marcos qui donne à son ami le mot de la fin pour son roman et l'énergie nécessaire pour l'achever (p. 301).

La quête d'un sens existentiel par la pratique autoréférentielle ne reste donc pas aporétique, puisqu'elle propose en dernière instance la refondation morale $\mathrm{du}$ rapport à autrui, à lire en contrepoint des actes et des tics narratifs du narrateur. C'est bien la superlativité même de l'autoréférence qui déclenche la prise de distance vis-à-vis de ce dernier, et permet d'investir une figure d'auteur implicite à partir de laquelle se reconstitue une autre réalité, une autre expérience de lecture. Georges Tyras fait apparaître que le sens de Soldados de Salamina tient à la centralité de l'autoréférence : l'enquête menée à bien par le narrateur ne vise pas tant à la reconstruction historique qu'à faire de la figure du personnage-écrivain la clef de voûte de l'édifice romanesque ${ }^{56}$. Or j'ai voulu démontrer, en analysant l'incidence de la formation progressive d'un lecteur implicite sur l'acte concret de ma compréhension du texte - comme la méthode du réalisme intentionnel y invite -, que La velocidad de la luz présente précisément l'envers de la survalorisation du processus de création au cœur de la trame narrative.

Durant la première étape de lecture, le réalisme de La velocidad de la luz - en tant qu'ensemble des dispositifs textuels qui favorisent la projection par le lecteur de sa réalité sur le monde du texte - dépendrait, outre le réinvestissement des codes réalistes spécifiques mis en place dans Soldados de Salamina, de la limitation maximale des espaces d'indétermination du sens. On l'a vu : la structure du récit, les procédés contradictoires de paralipses/paralepses et la figure omnipotente du narrateur le verrouillent. On touche là à une sorte d'autoritarisme du réalisme, que pointe du doigt explicitement El vano ayer et que Villanueva explique ainsi : "novela realista será la que ponga en juego todos los mecanismos de control interno -textual- de la respuesta para inducirla en aquella dirección y con aquel sentido $"^{57}$. Son efficacité tiendrait à une ferme direction du lecteur à travers le texte -par le biais du lecteur implicite, dont le lecteur empirique est censé mimer la réponse. La deuxième interprétation que j'ai avancée implique, comme la première, une coopération réaliste du lecteur (le sense-making de Fludernik), mais plutôt à travers les non-dits du récit ${ }^{58}$.

55. Rodney s'engage au Vietnam par devoir et accepte, en dépit de sa répulsion, de participer à un documentaire par loyauté envers un ancien combattant. Le portrait qui est fait de Jenny est celui d'une femme qui ne renonce jamais à son exigence morale et se charge inlassablement du sauvetage de Rodney. Enfin, Marcos n'abandonne pas son ami en détresse, le soutient et lui pardonne son ingratitude.

56. Georges Tyras, "Rafael Sánchez Mazas ou la sale mine du "récit réel”... ", op. cit., p. 66.

57. Darío Villanueva, op. cit., [édition 2004] p. 173.

58. "la obra no solo contiene indicaciones explícitas de cómo ha de ser leida, sino que con aquello que le falta-[...] sus indeterminaciones-, está provocando respuestas cooperativas de su destinatario ", 
J'ai tenté d'analyser la façon dont ces deux romans jouent, de manière bien distincte tant du point de vue des procédés esthétiques que de la démarche éthique, mais tous deux avec des dispositifs relevant de l'autoréférence, sur les modes de référentialité réaliste. Par référentialité j’ai entendu les mécanismes qui activent notre tendance, acquise ou innée, à la lecture réaliste, d'après la théorie du « réalisme intentionnel ». Les dispositifs autoréférentiels (métatextuels ou autofictionnels, entre autres) sont pourtant le plus souvent considérés comme anti-réalistes, car contredisant le critère de discrétion et d'impersonnalité de l'auteur, lié au principe réaliste de la lisibilité. Or, on a vu comment la configuration de l'auteur et du lecteur implicites servaient puissamment la productivité réaliste et l'évaluation éthique des romans.

Envisager l'efficacité avec laquelle un récit, plus que parler de la réalité, me fait parler de ma réalité, porte ses fruits auprès de récits contemporains pratiquant un "surcodage " réaliste $\mathrm{e}^{59}$ par insertion ou mimesis d'archives, puisant dans un type de référentialité extérieur au monde fictionnel. L'enjeu cognitif de la littérature est particulièrement mobilisé en Espagne dans le cadre de la "récupération de la mémoire historique " et de la crise sociale, politique et culturelle actuelle. À ce titre, l'approche théorique du réalisme que j'ai mise à profit pour travailler l'opérativité des notions de référence et autoréférence ne doit pas faire oublier les implications sociales et idéologiques que recouvre cette catégorie littéraire. Le cadre politique semble évacué ici chez Cercas, qui privilégie les ressorts plus traditionnels de la coopération du lecteur par l'identification et le pathos, mais en offrant un feuilleté, une progression ou un subtil va-et-vient entre lecture naïve et lecture critique au sein de chaque lecteur. Au contraire, se forge chez Rosa un méta-réalisme critique qui, en questionnant les façons dont le contexte artistique contemporain modèle notre interprétant, prend aussi valeur de formation citoyenne.

\section{Bibliographie}

Alsina Jean, "Référenciation, référentialisation - Los Bravos de Jesús Fernández Santos ", in Champeau Geneviève (sous la dir. de), Référence et autoréférence dans le roman espagnol contemporain, Actes du colloque international de Talence (1992), Bordeaux, Maison des Pays Ibériques, 1994, p. 51-64.

Booth Wayne, "Distance et point de vue ", in Barthes Roland et al. Poétique du récit, Paris, Seuil, 1977 [Poétique, 4, 1970], p. 85-113.

Carcelen Jean-François, «Écriture de l'obsession et obsession de l'écriture : Cerbero son las sombras, de Juan José Millás ", in Champeau Geneviève (sous la dir. de), Référence et autoréférence dans le roman espagnol contemporain, Actes du colloque international de Talence (1992), Bordeaux, Maison des Pays Ibériques, 1994, p. 173-185.

Ibid., p. 175.

59. Philippe Hamon, «Un discours contraint », op. cit., p. 139. 
Fludernik Monika, Towards a 'natural' narratology, London/New York, Routledge, 1996.

Genette Gérard, Discours du récit, Paris, Seuil, Essais, 2007 [1972].

- Fiction et Diction, Paris, Seuil, 2004 [1991].

Hamon Philippe, "Un discours contraint ", in Barthes Roland et al., Littérature et réalité, Paris, Seuil, 1982, [1973], p. 119-181.

- «Thème et effet de réel », Poétique, 64, 1985, p. 495-503.

Harshaw Benjamin, "Fictionality and Fields of Reference. Remarks on a theoretical framework ", Poetics Today, 5, 2, 1984, p. 227-251.

Herrnstein-Smith Barbara, On the margins of discourse, The University of Chicago Press, 1978.

Kindt Tom, "L' "auteur implicite”. Remarques à propos de l'évolution de la critique d'une notion entre narratologie et théorie de l'interprétation ", in Pier John (sous la dir. de), Théorie du récit. L'apport de la recherche allemande, Presses universitaires du Septentrion, 2007.

Lilti Antoine et Anheim Etienne, "Introduction ", in "Savoirs de la littérature ", Annales. Histoire, Sciences sociales, $\mathrm{n}^{\circ}$ 2, mars-avril 2010, p. 253-260.

Lyon-Caen Judith, La lecture et la vie, Taillandier, 2006.

- et Ribart, Dinah, L'historien et la littérature, Paris, La Découverte, 2010.

Paterson Janet, "L'Autoreprésentation : formes et discours ", in "L'autoreprésentation. Le texte et ses miroirs ", Texte, Revue de critique et de théorie littéraire, n 1,1982 , p. 177-195.

Phelan James, Experiencing fiction : judgments, progression and the rhetorical theory of narrative, Ohio State University Press, 2007.

Rebreyend Anne-Laure, "Deconstrucción del realismo. Paradojas de la metáfora del teatro en El vano ayer, de Isaac Rosa ", in Florenchie Amélie (sous la dir. de), "Últimas noticias del realismo en España », Pasavento, Revista de Estudios Hispánicos, vol. II, $n^{\circ} 1$, invierno 2014, p. 57-76.

Riffatterre Michael, "L'illusion référentielle ", in Barthes Roland et al., Littérature et réalité, Paris, Seuil, 1982 [1978], p. 91-118.

Ricœur Paul, Temps et récit 1, Paris, Seuil, 1983.

Román Román Isabel, «El juego con las expectativas del lector realista: la narrativa de la primera vanguardia española " in Fabry Geneviève et Canaparo Claudio (sous la dir. de), El enigma de lo real. Las fronteras del realismo en la narrativa del siglo XX, Oxford, Peter Lang, 2007.

Rosa Isaac, El vano ayer, Seix Barral, 2004.

Schaeffer Jean-Marie, Pourquoi la fiction?, Paris, Seuil, 1999.

Tyras Georges, "Rafael Sánchez Mazas ou la sale mine du récit réel (sur Les Soldats de Salamine, de Javier Cercas)», in Témoigner. Entre Histoire et Mémoire, Revue 
pluridisciplinaire de la fondation Auschwitz, éditions Kimé, nº 102, janvier-mars 2009.

Vila Juan, "Les fantômes (auto)référentiels du ciné Roxy ", in Champeau Geneviève (sous la dir. de), Référence et autoréférence dans le roman espagnol contemporain, Actes du colloque international de Talence (1992), Bordeaux, Maison des Pays Ibériques, 1994, p. 81-94.

Villanueva Darío, Teorías del realismo literario, Madrid, Biblioteca Nueva, 2004 [Instituto de España/Espasa Calpe, 1992]. 